\title{
Sexual Dimorphism in the Gut Microbiome
}

\author{
Clarissa Ren, Kristyn E. Sylvia \\ Faculty Mentor: Dr. Gregory Demas, Department of Biology, Indiana University Bloomington
}

\begin{abstract}
The gut microbiome has received increasing interest in past years due to its link to many diseases and its potential in therapy. One often-overlooked and newer area of research is the sexual dimorphism in the gut microbiome, and how it relates to the sex differences in behavior, diseases, and the underlying makeup between that of males and females. Reviewing the literature has demonstrated that in several organisms, adult males and females do naturally have different compositions of gut microbes. Differences between the sexes in gut microbiome have also been correlated with differences between the sexes in social behavior and various disorders. A thorough understanding of sexual dimorphism in the gut microbiome is crucial to designing better studies, understanding the mechanism of the diseases and behaviors tied to the sexual dimorphism in the microbiome, and fine-tuning more precise treatments that account for the sex of the individual.
\end{abstract}

KEYWORDS: microbiome, sex-specific, social behavior, endocrine system

\section{INTRODUCTION}

At

lthough century-old studies have been found examining the link between the microbiota and brain, this research was not recognized and was viewed with skepticism (Lyte and Cryan, 2014). A PubMed search of "gut microbiota" five years ago would have produced 3,180 publications, but as of April 2018, it produces 16,878 publications. By now, the Human Microbiome Project has characterized the gut microbiome in both diseased and healthy humans in a $\$ 173$ million project. The potential role of the gut microbiome in disease, as well as potential for application in drug therapies, has made the gut microbiome an increasingly studied topic.

Our gut microbes outnumber us at about 100 trillion microbial cells compared to our 10 trillion human cells (Whitman et al., 1998). Although we share 99.99 percent of our DNA with our fellow humans, our gut microbes may only be 10 percent similar to the next person's (Knight, 2014). Gut microbes have a wide range of functions from digesting food to metabolizing drugs, interacting with our immune and nervous system, and balancing our hormones (Knight, 2014). An unbalanced gut microbiome has been linked to a myriad of diseases including irritable bowel syndrome (IBS), inflammatory bowel disease (IBD), obesity, diabetes, multiple sclerosis, autism, allergic diseases, depression and anxiety, and more (Ghaisas et al., 2016). The correlational connection between gut microbiomes and human diseases has inspired a search for treatments ranging from diet to probiotics, prebiotics, and fecal transplanting (Vrieze et al., 2013). When understanding human diseases, animal studies cannot always be directly translated to humans and the results can sometimes contradict the results found in humans, but animal studies are nevertheless the start to better understanding the underlying mechanisms.

One often-overlooked aspect of the gut microbiome is how the sex of the individual influences it and how the microbiome communicates with the endocrine system to influence the sex of the individual (Org et al., 2016). Disparities have been observed in the incidence of specific diseases between men and women. For example, women have double the chance of experiencing depression or anxiety as men, while autism has a 4:1 ratio for men compared to women. (Fombonne, 2005; L.C. Klein, \& Corwin, 2002; Kornstein et al., 2000). Although the gut microbiome likely fuels a part of this disparity, there are also likely other factors such as societal differences between men and women when visiting the doctor (Pinkhasov et al., 2010). This review aims to summarize recent developments on sex differences in the gut microbiome as well as their relation to sex differences in a range of behaviors and disorders with therapy potential (Kozik et al., 2017; Zelinkova \& der Woude, 2014).

\section{METHODS}

This review was primarily written with the aid of PubMed. A search of "gut microbiome sex difference" on PubMed yielded a total of 37 publications. Studies that did not specifically focus on differences between the sexes and their relations to the gut microbiome were omitted. For example, one of the omitted publications was called "Prevalence of Small Intestinal Bacterial Overgrowth in Multiple Sclerosis: A Case-Control Study from China," and although this study does control for sex by comparing patients with and without multiple sclerosis of the same sex, it does not examine the differences between the sexes. Review articles were examined, but were also omitted as bases for this review. In addition to searches on sex differences, searches were also made on PubMed and Google Scholar in specific areas that needed more exploration, such as searches on more details of social behavior or the functions of specific bacteria.

\section{DIFFERENT STARTING GUT COMPOSITIONS BETWEEN SEXES}

Even before treatment, there are disparities in the gut microbiome between males and females. For example, Kozik et al. (2017) found that the gut microbiomes of four- to seven-week-old male mice have less species evenness and richness than those of female mice of a similar age. In addition, wild type (WT) male mice have significantly more Ruminococcaceae, Ruminococcus, and Anaerostipes, while WT female mice have significantly more Peptostreptococcaceae (Kozik et al., 2017). Anaerostipes is known to produce butyric acid, the primary energy source of cells in the colon (Donohoe et al., 2011; Kant, Rasinkangas, Satokari, Pietilä, \& Palva, 2015). Ruminococcaceae and Ruminococcus are also involved in the fermentation of carbohydrates and are strictly anaerobic (Vos et al., 2009). Meanwhile, Peptostreptococcaceae has been found to metabolize more protein and amino acids than carbohydrates (Vos et al., 2009). These differences may translate to downstream differences in behavior and physiology because different metabolic reactants 
and products will interact differently with the endocrine, nervous, and immune system.

Another study showed that adult C57BL/6J mice had differently composed gut microbiomes between the sexes. While males had more Bacteroidia and Erysipelotrichi, females had more Clostridia and Mollicutes (Davis et al., 2017). Interestingly enough, female C57BL/6J mice were found to have more Ruminococcaceae and Ruminococcus, which differs from the results found in wild-type mice by Kozik et al. This indicates that there are many other factors besides sex that also influence the gut microbiome, such as type of mice or diet. However, with all confounding variables accounted for, sex differences are often still found to be present within the same species and conditions.

Broiler chickens were also found to have different starting gut microbiomes between the sexes-while males had more Bacteroides, females had more Clostridium and Shigella (Lee et al., 2017). Lee et al. speculates that this difference may be related to the differing growth potentials between male and female broiler chicken. Therefore, some bacterial differences are likely responsible for traits associated with the sex, such as larger or smaller size.

\section{GUT MICROBIOME SEXUAL DIMORPHISM IN SOCIAL BEHAVIOR}

Differences between sexes with relation to the gut microbiome can lead to different social behaviors (Sylvia et al., 2017). In rats prenatally exposed to prenatal propionic acid (PPA), a short chain fatty acid produced by many of the antibiotic-resistant enteric bacteria in the gut, both males and females had impaired nest-seeking response, but males moved towards a novel object more and had higher locomotor activity than females (Foley et al., 2014). Although this study did not examine the specific gut microbiome composition in males and females, it did show that having the same relative amount of a gut bacterial product had different effects between the sexes (Foley et al., 2014). This has important implications because PPA is an environmental factor that has been associated with autism spectrum disorder (Macfabe, 2012), suggesting that social behavior disorders related to the autism spectrum should be studied with attention to sex-specificity.

The gut microbiome can also respond differently between the sexes to treatments for anxiety and depressive-like behaviors. Davis et al. (2017) socially isolated male and female mice, then treated them with docasahexaenoic acid (DHA), a long chain fatty acid known to decrease anxiety and depressive symptoms. Treatment with DHA affected the gut microbiome of males, but not females (Davis et al., 2017). Socially isolated males showed much more anxiety and depressive behavior, such as entering the open arms of a maze more times or having a greater sucrose preference (Davis et al., 2017). In males, there was a negative correlation between the amount of Ruminococcus and increased anxiety and anhedonia-related behaviors, and a significant positive correlation between Allobaculum and decreased anxiety and anhedoniarelated behaviors (Davis et al., 2017). It is speculated that these sex differences may be due to differences in fatty acid metabolism (Davis et al., 2017). PICRUSt, a type of bioinformatics analysis that can determine the metagenome function of genetic information, showed that for control males, the KEGG pathway, including tryptophan biosynthesis, was enhanced, while fatty acid metabolism was enhanced for females (Davis et. al. 2017). The increased tryptophan can act as a precursor to serotonin and kynurenine while also increasing quinolinic acid levels, and these compounds can act on the central nervous system (Davis et al., 2017; Fukui,
Schwarcz, Rapoport, Takada, \& Smith, 1991; Schwarcz, Bruno, Muchowski, \& Wu, 2012; Udenfriend, Weissbach, \& Bogdanski, 1957). The kynurenine and quinolinic acid levels correspond to the increased level of anxiety-related behaviors (Maes, Ringel, Kubera, Berk, \& Rybakowski, 2012; Orlikov \& Ryzov, 1991).

Finally, aggression as a social behavior can also be differentially affected between sexes in Siberian hamsters treated with antibiotics (Sylvia, Jewell, Rendon, St. John \& Demas, 2017). Prior to antibiotic treatment, male and female Siberian hamsters had different gut microbiome compositions with male gut microbiome being composed of $\sim 50$ percent Firmicutes and $\sim 40$ percent Bacteroidetes and female gut microbiome being composed of $\sim 50$ percent Firmicutes and $\sim 30 \%$ Bacteroidetes. Antibiotic treatment in males led to changes in five phyla for males (Cyanobacteria, Elusimicrobia, Euyarchaeota, Proteobacteria, and TM7), whereas antibiotic treatment in females led to changes in three phyla (Cyanobacteria, Proteobacteria, and Tenericutes) (Sylvia et al., 2017). Furthermore, when given two treatments of antibiotic with a recovery phase, aggression decreased, but then returned to normal levels during recovery in male Siberian hamsters, whereas female Siberian hamsters showed a decrease in aggression that did not return to normal levels during recovery (Sylvia KE et al., 2017). The level of aggression was associated with the levels of Tenericutes, Cyanobacteria, and TM7 (Sylvia K.E. et al., 2017). Proposed mechanisms include a direct link between the gut microbiota and the central nervous system (CNS), as well as changes to the immune system cytokines that may influence the communication between the gut microbiome and brain (Sylvia K.E. et al., 2017). This study may provide a better understanding of the higher level of aggression observed in human men with depression than women with the same severity of depression (Martin et al., 2013).

\section{GUT MICROBIOME SEXUAL DIMORPHISM IN DISORDERS}

Sex differences in the gut microbiome play a role in colitis, which includes inflammatory bowel diseases like Crohn's disease (Kozik et al., 2017). Kozik et al. (2017) induced colitis in WT and tumor necrosis factor lacking mice $\left(\mathrm{Tnf}^{-/-}\right)$through treatment with 2,4,6-trinitrobenzenesulfonic acid (TNBS). Male WT mice were found to have significantly more severe colitis than female WT and Tnf ${ }^{-/}$mice of both sexes (Kozik et al., 2017). This more severe colitis in male WT mice was associated with higher levels of Alphaproteobacteria and other Proteobacteria, which have been associated with Crohn's disease in humans (Kozik et al., 2017; Shin, Whon, \& Bae, 2015). In humans, some studies assert that women are slightly more likely to develop IBD, while other studies found no difference between the sexes (Zelinkova and der Woude, 2014). The reason for these sex-specific differences are not known; however, researchers believe it may be influenced by hormones or interactions with environmental factors (Fairweather et al., 2008; Girón-González et al., 2000; S.L. Klein \& Flanagan, 2016; Trigunaite et al., 2015; Verthelyi \& Klinman, 2000).

Sex differences play a role in many autoimmune diseases like Type 1 diabetes. Although specific pathogen-free non-obese diabetic female mice have a 1.3- to 4.4-times higher rate of Type 1 diabetes than males, this sex-difference is lost in germ-free mice (Yurkovetskiy et al., 2013). Another study found that transferring a non-obese diabetic male mice's gut microbiota to a non-obese diabetic female mice's gut microbiota resulted in protection from Type 1 diabetes and a decrease in pancreatic islet inflammation and autoantibody production (Markle et al., 2013). 


\section{SUBSTANCES ALTER THE GUT MICROBIOME IN A SEX-SPECIFIC MANNER}

An excess of manganese (Mn) can induce Parkinson's disease (Chi, Gao, Bian, Tu, Ru, \& Lu, 2017) . When C57BL/6 mice were dosed with $\mathrm{MnCl}_{2}$, male mice showed an increase in Firmicutes whereas female mice showed a decrease in Firmicutes (Chi et al., 2017). In addition, Bacterodetes decreased significantly in male mice only (Chi et al., 2017). Several mechanisms were investigated, such as exposure to Mn's effect on oxidative stress, iron absorption, $\mathrm{Mn}^{2+}$ availability in the gastrointestinal tract, and quorum sensing affecting selective growth of bacteria in the gut (Chi et al., 2017). In addition to sex-specific differences in the gut microbiome, Chi et al. also found many other sex-specific differences. Bacterial genes in the lipopolysaccharide and DNA repair pathway were found to be up-regulated for females and down-regulated for males (Chi et al., 2017). The gene encoding for tryptophan synthesis was downregulated for females and up-regulated for males (Chi et al., 2017). The gene encoding for glutamate decarboxylase, which synthesizes GABA, was down-regulated in males only (Chi et al., 2017). Finally, fecal metabolites, an important component to the gut microbes and host communication, were found to be mostly down-regulated for males, but some were down-regulated and others were up-regulated for females (Chi et al., 2017). In the future, work should be done to investigate if there is any relationship between the sex-specific differences seen in the gut microbiome and the sex-specific differences seen in lipopolysaccharide and DNA repair, tryptophan and GABA synthesis, and fecal metabolite regulation. This may help pinpoint possible solutions for neurological disorders like Parkinson's disease.

Another substance that alters the gut microbiome in a sex-specific manner is nicotine (Chi, Mahbub, Gao, Bian, Tu, Ru \& Lu, 2017). Nicotine can influence human behavior by directly passing through the blood-brain barrier, affecting the reward system in the brain by triggering dopamine release, and regulating many neurotransmitters involved in learning, memory, and cognition (Pogun \& Yararbas, 2009; Rezvani \& Levin, 2001; Singer et al., 2004; Toth, Sershen, Hashim, Vizi \& Lajtha, 1992; Yu \& Wecker, 1994). When C57BL/6J mice were exposed to nicotine, male mice showed an increase in F16, Turicicibacteraceae, and Peptococcaceae, Christensenellaceae and a decrease in Dehalobacteriaceae (Chi, Mahbub et al., 2017). On the other hand, female C57BL/6J mice exposed to nicotine showed a decrease in F16, Christensenellaceae, and Anaeroplasmataceae (Chi, Mahbub et al., 2017). In addition, male mice decreased in weight whereas females did not (Chi, Mahbub et al., 2017). This may be explained by differences in the effect of nicotine on carbohydrate metabolism with the acetate synthesis genes up-regulated for males and the butyrate synthesis genes up-regulated for females (Chi, Mahbub et al., 2017). These body mass differences could be due in part to Christensenallaceae, which was associated with low body weight (Fischbach \& Segre, 2016). Furthermore, butyrate, which is up-regulated in females, is a major energy source for intestinal epithelial cells while acetate, which is down-regulated in males, is an appetite suppressor (Frost et al., 2014; Hague, Singh, \& Paraskeva, 1997). Another difference is increased oxidative stress and DNA repair gene expression in males, but not females (Chi, Mahbub et al., 2017). Finally, many amino acids were differentially influenced by nicotine between the sexes, and these amino acids are often neurotransmitters or precursors of neurotransmitter (Chi, Mahbub et al., 2017). Although future work must be done to determine if the gut microbiome is the change agent for the other differences, one can see already that males and females can react to the same treatment in largely disparate ways.

\section{POTENTIAL MECHANISMS IN GUT MICROBIOME SEX DIFFERENCES}

One of the major differences between a male and a female is the different balance of hormones, which has often been hypothesized as a factor in the sexual dimorphism of the gut microbiome. Therefore, it is only natural that some studies would focus specifically on the link between sex differences in hormones and the gut microbiome.

Several studies have demonstrated that colonization with certain microbiota in germ free, non-obese, diabetic mice can cause changes in hormone levels. Female microbiota are found to be more similar to prepubescent microbiota than male microbiota (Yurkovetskiy et al., 2013). Castration of male mice makes their gut microbiome more similar to that of a female, indicating that it is hormones, rather than sex by XX or XY chromosomes, that determine the gut microbiome (Yurkovetskiy et al., 2013). Not only do the hormones affect the gut microbiome, but the gut microbiome also affects hormones. Transferring an adult male's gut microbiota to an immature female's gut microbiota changed the gut microbiota, increased testosterone levels, and left metabolomic changes in the female recipient (Markle et al., 2013).

To examine how sex hormones affect the gut microbiome, Org et al. performed a gonadectomy on 3 strains of mice fed chow or high fat diets (2016). Across all three strains, Ruminococcacea was found to be significantly different in control males and gonadectomy males (Org et al., 2016). Furthermore, when given testosterone following gonadectomy, significant changes were no longer found in two of the strains of male mice (Org et al., 2016). In females, Akkermansia was found to be less in gonadectomy females than control females on high fat diet in two strains (Org et al., 2016). Finally, bile acid levels were greater in gonadectomy males than females under a high fat diet, and bile acids can also affect the gut microbiome (Islam et al., 2011; Li \& Chiang, 2015; Org et al., 2016).

\section{FUTURE DIRECTIONS}

Over the past few years, research has shed light on the sex dependence of the gut microbiome in areas including social behavior, neurological disorders, reproduction, bowel diseases, autoimmune diseases, and hormonal influences. More areas will likely still be discovered because it is easy to miss the sex differences when confounded with genetic and environmental factors; for example, Org et al. found sex differences when examining within a strain of mice, but these differences were obscured when examined across three strains of mice (2016). Given the ease of missing a sex difference, future researchers should keep the sex of the subject in mind when designing an experiment to avoid confounding the research.

Researchers must also pay attention to what type of model organism they are using and be wary of making broad generalizations. For example, different strains of mice have different starting compositions between the sexes-for C57BL/6J mice, females had more abundant Ruminococcaceae and Ruminococcus, while in wild type mice, males had more abundant Ruminococcaceae and Ruminococcus (Davis et al., 2017; Kozik et al., 2017). How much of this difference is due to inherent sex differences, and how much of this difference is due to the interaction of sex differences with other factors? Furthermore, researchers must have a comprehensive understanding of the starting gut composition of their model organism because it can often differ from what is expected in a human. For example, male mice were found to be more likely to develop colitis, but colitis is more likely to be associated with women or have no difference between genders in humans (Kozik et al., 2017, Zelinkova \& der Woude, 2014). 
Future research should also focus more on the mechanisms behind these sex differences as well as causality versus correlation. Is the gut microbiome what changes first to cause differences in social behavior, or does another factor that alters the social behavior also change the gut microbiome in a sex-specific manner? Although several studies have proposed mechanisms related to sex differences in hormones, the CNS, and the immune response, most of the above studies have not found a direct answer for the mechanism. Pinpointing a detailed mechanism will not only allow for a better understanding of the disease, but will also allow better ways to look for a treatment for the various gut microbiomerelated diseases.

Finally, sex difference in the gut microbiome will be an important consideration when looking at treatments. Given the differences in gut microbiome between the sexes, researchers will need to figure out how to fine-tune the treatment, whether it is through diet, probiotics, or fecal transplanting, in order to get the best results. In addition, when synthesizing a treatment, one must consider how multiple factors including sex, age, body weight, and diet all come together to create a dynamic and individualistic gut microbiome. Considerations like these allow researchers to be able to better design studies and therapies.

\section{AUTHOR INFORMATION}

All correspondence should be sent to the first author:

clarissa.ren@gmail.com

\section{REFERENCES}

Chi, L., Gao, B., Bian, X., Tu, P., Ru, H., \& Lu, K. (2017).

Manganese-induced sex-specific gut microbiome perturbations in C57BL/6 mice. Toxicology and Applied Pharmacology, 331, 142-153.

Chi, L., Mahbub, R., Gao, B., Bian, X., Tu, P., Ru, H., \& Lu, K. (2017). Nicotine Alters the Gut Microbiome and Metabolites of Gut-Brain Interactions in a Sex-Specific Manner. Chemical Research in Toxicology, 30(12), 2110-2119.

Davis, D.J., Hecht, P.M., Jasarevic, E., Beversdorf, D.Q., Will, M.J., Fritsche, K., \& Gillespie, C.H. (2017). Sex-specific effects of docosahexaenoic acid (DHA) on the microbiome and behavior of socially-isolated mice. Brain, Behavior, and Immunity, 59, 38-48.

Donohoe, D.R., Garge, N., Zhang, X., Sun, W., O’Connell, T.M., Bunger, M.K., \& Bultman, S.J. (2011). The microbiome and butyrate regulate energy metabolism and autophagy in the mammalian colon. Cell Metabolism, 13(5), 517-526.

Fairweather, D., Frisancho-Kiss, S., \& Rose, N.R. (2008). Sex Differences in Autoimmune Disease from a Pathological Perspective. The American Journal of Pathology, 173(3), 600-609.

Fischbach, M.A., \& Segre, J.A. (2016). Signaling in HostAssociated Microbial Communities. Cell, 164(6), 1288-1300.

Foley, K.A., MacFabe, D.F., Vaz, A., Ossenkopp, K.P., \& Kavaliers, M. (2014). Sexually dimorphic effects of prenatal exposure to propionic acid and lipopolysaccharide on social behavior in neonatal, adolescent, and adult rats: Implications for autism spectrum disorders. International Journal of Developmental Neuroscience, 39, 68-78.
Fombonne, E. (2005). Epidemiology of autistic disorder and other pervasive developmental disorders. The Journal of Clinical Psychiatry, 66 Suppl. 10, 3-8.

Frost, G., Sleeth, M.L., Sahuri-Arisoylu, M., Lizarbe, B., Cerdan, S., Brody, L., \& Bell, J.D. (2014). The short-chain fatty acid acetate reduces appetite via a central homeostatic mechanism. Nature Communications, 5, 3611.

Fukui, S., Schwarcz, R., Rapoport, S.I., Takada, Y., \& Smith, Q.R. (1991). Blood-brain barrier transport of kynurenines: implications for brain synthesis and metabolism. Journal of Neurochemistry, 56(6), 2007-2017.

Ghaisas, S., Maher, J., \& Kanthasamy, A. (2016). Gut microbiome in health and disease: linking the microbiome-gut-brain axis and environemental factors in the pathogenesis of systemic and neurodegenerative diseases. Pharmacology \& Therapeutics, 158, 52-62.

Girón-González, J.A., Moral, F.J., Elvira, J., García-Gil, D., Guerrero, F., Gavilán, I., \& Escobar, L. (2000). Consistent production of a higher TH1:TH2 cytokine ratio by stimulated $\mathrm{T}$ cells in men compared with women. European Journal of Endocrinology, 143(1), 31-36.

Hague, A., Singh, B., \& Paraskeva, C. (1997). Butyrate acts as a survival factor for colonic epithelial cells: further fuel for the in vivo versus in vitro debate. Gastroenterology, 112(3), 1036-1040.

Islam, K.B.M.S., Fukiya, S., Hagio, M., Fujii, N., Ishizuka, S., Ooka, T., \& Yokota, A. (2011). Bile Acid Is a Host Factor That Regulates the Composition of the Cecal Microbiota in Rats. Gastroenterology, 141(5), 1773-1781.

Kant, R., Rasinkangas, P., Satokari, R., Pietilä, T.E., \& Palva, A. (2015). Genome Sequence of the Butyrate-Producing Anaerobic Bacterium Anaerostipes hadrus PEL 85. Genome Announcements, 3(2), e00224-15.

Klein, L.C., \& Corwin, E.J. (2002). Seeing the unexpected: how sex differences in stress responses may provide a new perspective on the manifestation of psychiatric disorders. Current Psychiatry Reports, 4(6), 441-448.

Klein, S.L., \& Flanagan, K.L. (2016). Sex differences in immune responses. Nature Reviews Immunology, 16(10), 626-638.

Kornstein, S.G., Schatzberg, A.F., Thase, M.E., Yonkers, K.A., McCullough, J.P., Keitner, G.I., \& Keller, M.B. (2000). Gender differences in chronic major and double depression. Journal of Affective Disorders, 60(1), 1-11.

Kozik, A.J., Nakatsu, C.H., Chun, H., \& Jones-Hall, Y.L. (2017). Age, sex, and TNF associated differences in the gut microbiota of mice and their impact on acute TNBS colitis. Experimental and Molecular Pathology, 103(3), 311-319.

Lee, K.C., Kil, D.Y., \& Sul, W.J. (2017). Cecal microbiome divergence of broiler chickens by sex and body weight. Journal of Microbiology (Seoul, Korea), 55(12), 939-945.

Li, T. \& Chiang, J.Y.L. (2015). Bile acids as metabolic regulators. Current Opinion in Gastroenterology, 31(2), 159-165.

Macfabe, D.F. (2012). Short-chain fatty acid fermentation products of the gut microbiome: implications in autism spectrum disorders. Microbial Ecology in Health and Disease, 23. 
Maes, M., Ringel, K., Kubera, M., Berk, M., \& Rybakowski, J. (2012). Increased autoimmune activity against 5-HT: a key component of depression that is associated with inflammation and activation of cell-mediated immunity, and with severity and staging of depression. Journal of Affective Disorders, 136(3), 386-392.

Markle, J.G.M., Frank, D.N., Mortin-Toth, S., Robertson, C.E., Feazel, L.M., Rolle-Kampczyk, U., \& Danska, J.S. (2013). Sex Differences in the Gut Microbiome Drive Hormone-Dependent Regulation of Autoimmunity. Science, 339(6123), 1084-1088.

Martin, L.A., Neighbors, H.W., \& Griffith, D.M. (2013). The experience of symptoms of depression in men vs. women: analysis of the National Comorbidity Survey Replication. JAMA Psychiatry, 70(10), 1100-1106.

Org, E., Mehrabian, M., Parks, B. W., Shipkova, P., Liu, X., Drake, T.A., \& Lusis, A.J. (2016). Sex differences and hormonal effects on gut microbiota composition in mice. Gut Microbes, 7(4), 313-322.

Orlikov, A. \& Ryzov, I. (1991). Caffeine-induced anxiety and increase of kynurenine concentration in plasma of healthy subjects: a pilot study. Biological Psychiatry, 29(4), 391-396.

Pinkhasov, R.M., Wong, J., Kashanian, J., Lee, M., Samadi, D., Pinkhasov, M.M., \& Shabsigh, R. (2010). Are men shortchanged on health? Perspective on health care utilization and health risk behavior in men and women in the United States. The International Journal of Clinical Practice, 64(4), 457-487.

Pogun, S. \& Yararbas, G. (2009). Sex Differences in Nicotine Action. In Nicotine Psychopharmacology (pp. 261-291). Berlin, Heidelberg: Springer Berlin Heidelberg.

Rezvani, A.H., \& Levin, E.D. (2001). Cognitive effects of nicotine. Biological Psychiatry, 49(3), 258-267.

Schwarcz, R., Bruno, J.P., Muchowski, P.J., \& Wu, H.Q. (2012). Kynurenines in the mammalian brain: when physiology meets pathology. Nature Reviews Neuroscience, 13(7), 465-477.

Shin, N.R., Whon, T.W., \& Bae, J.W. (2015). Proteobacteria: microbial signature of dysbiosis in gut microbiota. Trends in Biotechnology, 33(9), 496-503.

Singer, S., Rossi, S., Verzosa, S., Hashim, A., Lonow, R., Cooper, T., \& Lajtha, A. (2004). Nicotine-induced changes in neurotransmitter levels in brain areas associated with cognitive function. Neurochemical Research, 29(9), 1779-1792.

Sylvia, K.E., Jewell, C.P., Rendon, N.M., St. John, E.A., \& Demas, G.E. (2017). Sex-specific modulation of the gut microbiome and behavior in Siberian hamsters. Brain, Behavior, and Immunity, 60, 51-62.

Toth, E., Sershen, H., Hashim, A., Vizi, E.S., \& Lajtha, A. (1992). Effect of nicotine on extracellular levels of neurotransmitters assessed by microdialysis in various brain regions: role of glutamic acid. Neurochemical Research, 17(3), 265-271.

Trigunaite, A., Dimo, J., \& Jørgensen, T.N. (2015). Suppressive effects of androgens on the immune system. Cellular Immunology, 294(2), 87-94.

Udenfriend, S., Weissbach, H., \& Bogdanski, D.F. (1957). Biochemical findings relating to the action of serotonin. Annals of the New York Academy of Sciences, 66(3), 602-608.
Verthelyi, D., \& Klinman, D.M. (2000). Sex hormone levels correlate with the activity of cytokine-secreting cells in vivo. Immunology, 100(3), 384-390.

Vos, P., Garrity, G., Jones, D., Krieg, N., Ludwig, W., Rainey, F., Schleifer, K., \& Whitman, W. (2009). Bergey's Manual of Systematic Bacteriology: Volume 3: The Firmicutes. Springer.

Vrieze, A., de Groot, P.F., Kootte, R.S., Knaapen, M., Nood, E., Nieuwdorp, M (2013). Fecal transplant: A safe and sustainable clinical therapy for restoring intestinal microbial balance in human disease? Best Practice \& Research Clinical Gastroenterology, 27(1), 127-137.

Whitman, W.B., Coleman, D.C., \& Wiebe, W.J. (1998). Prokaryotes: the unseen majority. Proceedings of the National Academy of Sciences of the United States of America, 95(12), 6578-6583.

Yu, Z.J. \& Wecker, L. (1994). Chronic nicotine administration differentially affects neurotransmitter release from rat striatal slices. Journal of Neurochemistry, 63(1), 186-194.

Yurkovetskiy, L., Burrows, M., Khan, A.A., Graham, L., Volchkov, P., Becker, L. \& Chervonsky, A.A. (2013). Gender Bias in Autoimmunity Is Influenced by Microbiota. Immunity, 39(2), 400-412.

Zelinkova, Z. \& der Woude, J. (2014). Gender and Inflammatory Bowel Disease. Journal of Clinical Cell Immunology, 5(4). 